\title{
NON-MHC ANTIGENS AND THEIR RELATIVE RESISTANCE TO IMMUNOSUPPRESSION AFTER CORNEAL TRANSPLANTATION
}

\author{
SUSAN M. NICHOLLS ${ }^{1}$, BENJAMIN A. BRADLEY ${ }^{2}$ and DAVID L. EASTY ${ }^{3}$ \\ Bristol
}

\begin{abstract}
SUMMARY
We have used a high responder rat model to examine the role that non-MHC antigens play in corneal graft rejection. Recipients were backcross animals derived from a cross between two inbred strains, which mimicked the human outbred population in that donor and recipient could be matched or mismatched for MHC antigens, while non-MHC mismatches were variable and unknown. All mismatched grafts and $87 \%$ of matched grafts were rejected (median survival 11 and 17 days respectively). The high incidence of rejection of matched grafts indicates that several independently segregating non-MHC genes play a role in rejection. Moreover, the immune response to matched grafts appeared resistant to immunosuppression, suggesting that matching does not permit reduced dosage of immunosuppressants. A mechanism is discussed whereby matching at the class II locus may enhance presentation of mismatched histocompatibility antigens or viral peptides derived from infected graft cells, thereby prejudicing graft survival.
\end{abstract}

Despite numerous studies since the early $1970 \mathrm{~s}^{1-10}$ the precise value of major histocompatibility complex (MHC) matching in corneal transplantation is still not fully established, even in high-risk cases, although for other organs such as kidney and heart, matching is clearly beneficial. Conflicting results, particularly with respect to class II (HLA-DR) matching, have undoubtedly stemmed from many differences in study design (e.g. prospective versus retrospective, whether or not high-risk cases have

From: ${ }^{1}$ University of Bristol, Department of Ophthalmology, School of Medical Sciences; ${ }^{2}$ University of Bristol, Department of Transplantation Sciences, Professional Unit, Southmead Health Services; ${ }^{3}$ University of Bristol, Department of Ophthalmology, Bristol Eye Hospital, Bristol, UK.

Correspondence to: Susan Nicholls, PhD, Department of Ophthalmology, School of Medical Sciences, University Walk, Bristol BS8 1TD, UK. been considered separately), differences in selection criteria and in treatment protocols. In general class I (HLA-A and -B) matching appears beneficial, although in the recent US Collaborative Corneal Transplantation Studies (CCTS), involving aggressive immunosuppression, no benefit of HLA matching was evident during the 2 year follow-up period (although ABO matching appeared advantageous). DR matching has been claimed to be both beneficial ${ }^{8}$ and detrimental. ${ }^{10}$

There remain a number of questions to be answered. It is said that $70 \%$ of rejection episodes occur within the first year, ${ }^{9,11,12}$ but studies of graft outcome are frequently concluded after 1 or 2 years. The effect of matching on long-term survival has not been considered, although rejection can occur as long as 15 years after transplantation..$^{13}$ Matching in humans is performed against a background of variable non-MHC mismatches. These, or other unexplored factors such as infectious agents, might contribute significantly to human graft rejection, particularly late-onset rejection. If non-MHC antigens are important, they may explain inconsistent results of matching studies and affect future matching practices. Moreover, heavy immunosuppression, as given in the CCTS, may induce serious ocular sideeffects. We therefore need to address the question of whether or not matching permits lower doses of immunosuppression to be given.

What can be learnt about matching from animal models? The rat is a useful experimental animal because corneal transplantation is both technically feasible and yields reproducible results, while inbred strains of defined MHC type are available. Thus, donor and recipient can be matched for background genes and the effect of isolated MHC class I or II disparities can be examined. Conversely, the effect of non-MHC disparities can be assessed with precision by matching donor and recipient across the entire 
MHC complex. In humans, this cannot be done because non-MHC mismatches are supplemented by those of the less polymorphic MHC loci - HLA-C, -DP and -DQ - which are not included in matching protocols.

Since the first successful transplantation model in the rat was reported in $1985,{ }^{14}$ many studies have been performed in different laboratories using a large number of different strain combinations. Without immunosuppressive treatment, survival of grafts between strains mismatched across the entire MHC varies according to the strain combination from a median of 7.5 days $\left(\mathrm{BN}\left[\mathrm{RT} 1^{\mathrm{n}}\right]\right.$ to PVG $\left[\mathrm{RT}^{\mathrm{c}}\right]$ ) to $>100$ days (AUG $\left[\mathrm{RT} 1^{\mathrm{c}}\right]$ to WAG[RT1 $\left.\left.{ }^{\mathrm{u}}\right]\right) .{ }^{15}$ While results may be influenced to some extent by differences in transplantation procedures in different laboratories (e.g. the use of continuous as opposed to interrupted sutures or whether or not sutures are removed), they are consistent in that matching for class I is more critical than matching for class II. Depending on the strain combinations class I disparity alone induces an $18 \%{ }^{16}$ or a $58 \%{ }^{17}$ incidence of rejection in naive hosts, while class II incompatibility alone does not provoke rejection ${ }^{18,}{ }_{19}$ unless the recipient is preimmunised. ${ }^{19}$ These results are consistent with the human situation in that HLA-A/B matching appears to be beneficial but matching for HLA-DR may be detrimental. $^{10}$

Rat studies also show that non-MHC antigens are important targets for rejection. Katami et al., ${ }^{17}$ using congenic rat strains, observed a $48 \%$ incidence of rejection of MHC-matched grafts (DA [RT1 $\left.{ }^{\mathrm{a}}\right]$ to PVG.RT1 ${ }^{\text {a }}$ ) compared with $85 \%$ between fully mismatched strains (DA to PVG). Rejection in matched grafts was later in onset.

To explore in more detail the role of non-MHC

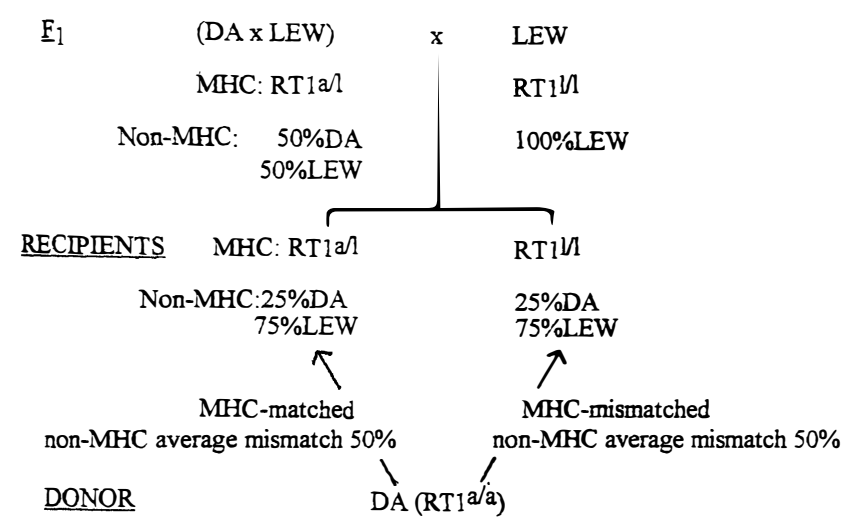

Fig. 1. Rat model designed to mimic human outbred population. Backcross $(D A \times L E W) F_{1} \times L E W$ recipients of $R T 1^{\text {a/l }}$ or $R T^{\text {all }}$ haplotype were either MHC-matched or $M H C$-mismatched with inbred $D A\left(R T 1^{a / a}\right)$ donors. The ratio of $D A: L E W$ non-MHC genes in recipients was variable, but averaged $25 \% D A$, i.e. half a haploid genome. Thus, the non-MHC disparity averaged 50\%. antigens we have tried to mimic the human outbred situation more closely using a backcrossed hybrid model derived from two inbred strains. ${ }^{20}$ DA $\times$ $\mathrm{LEW}\left(\mathrm{RT} 1^{1}\right)$ rats were backcrossed to LEW and the offspring received female DA strain corneal grafts. The DA and LEW strains differ for an entire MHC haplotype and at multiple non-MHC loci and represent a strongly rejecting strain combination. Use of backcross animals as recipients simulates the human situation better than wholly inbred or congenic models in that grafts can be either matched or mismatched for MHC against a background of non-MHC mismatches that, as with humans, are variable for each donor-recipient pair. Recipients (either $\mathrm{RT}^{\mathrm{a} / 1}$ or $\mathrm{RT} 1^{1 / 1}$, identified by indirect haemagglutination assay ${ }^{20}$ ) were either MHCmatched or mismatched with the donor, while nonMHC disparity varied theoretically from 0 to $100 \%$, averaging $50 \%$ (Fig. 1). A high-risk situation was provoked by leaving sutures in place. Grafts were examined daily for 3 days, then four times weekly for signs of rejection (opacity, oedema and vascularisation). If rejection had not occurred by day 50 , examination was reduced to two to three times weekly until at least day 100 . The day of rejection was defined as the day when central donor corneal opacity and oedema became moderate or severe.

In a total of 77 such transplants performed without immunosuppressive therapy, matching was beneficial ( $p=0.00002$; Fig. 2) but there was a high incidence of rejection of matched grafts, $87 \%$ of which were ultimately rejected. The clinical signs of rejection

\section{Percent survival}

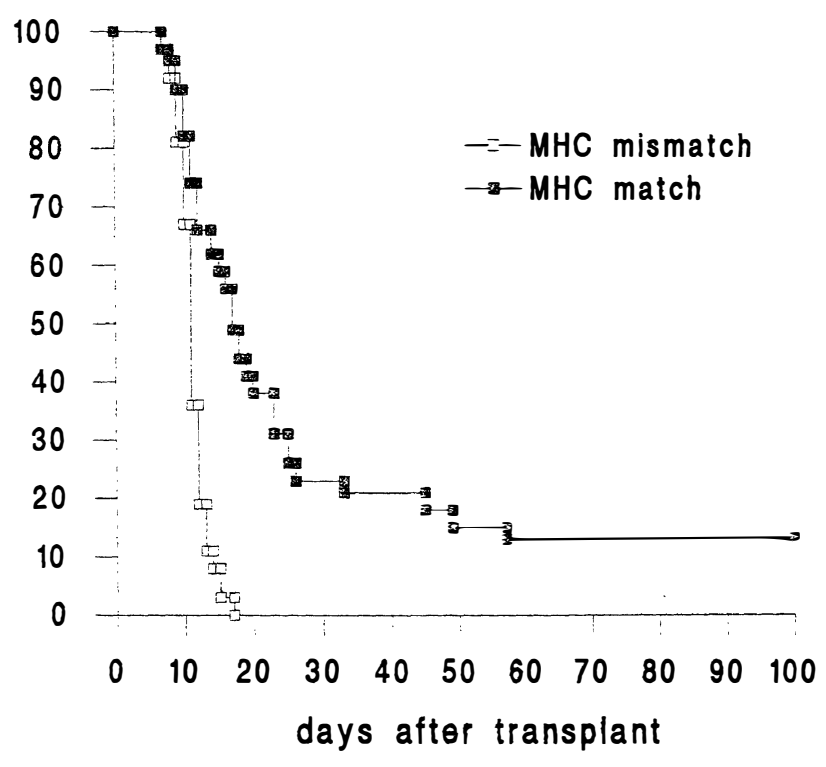

Fig. 2. Survival of 77 rat penetrating corneal grafts performed without immunosuppression, half of which were MHC-matched and half MHC-mismatched. Matching improved graft survival ( $\mathrm{p}=0.00002$, Mann-Whitney Utest), but $87 \%$ of matched grafts were ultimately rejected. 


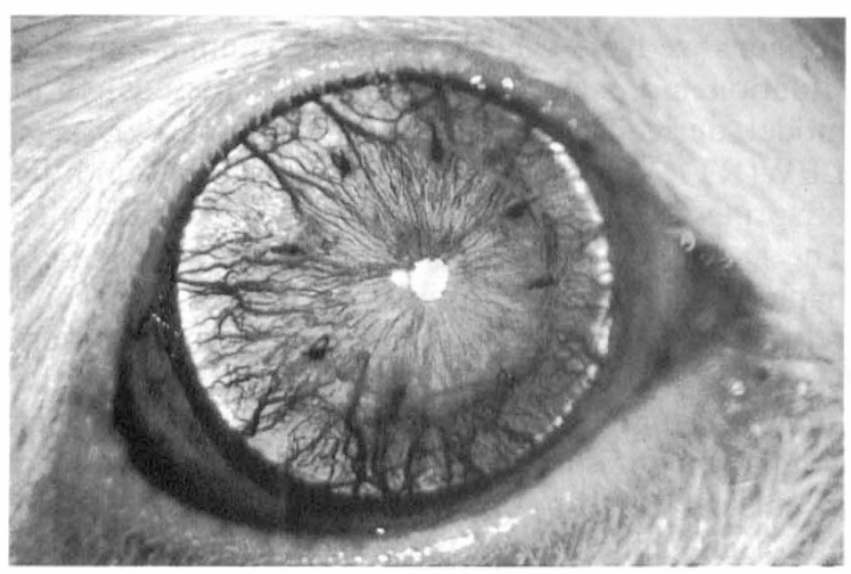

(a)

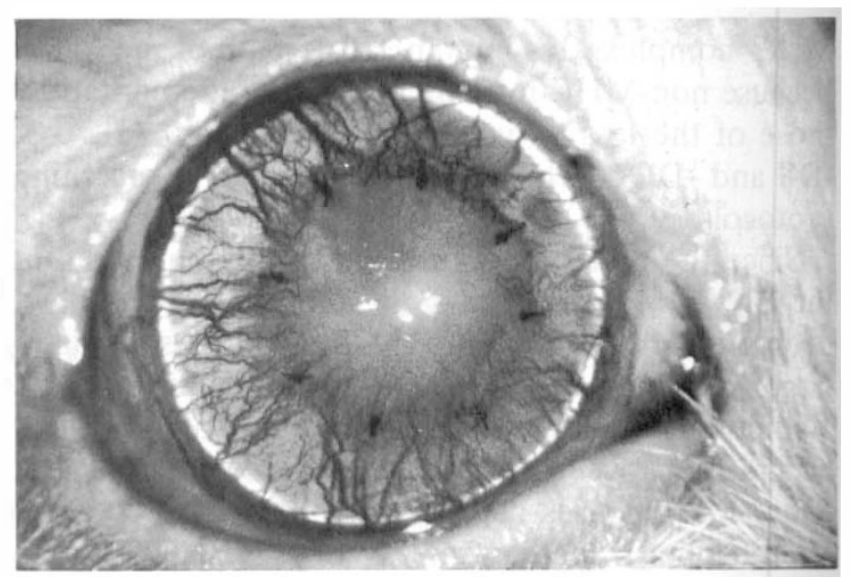

(b)

Fig. 3. Clinical rejection of an MHC-mismatched corneal graft in an albino rat. No immunosuppression was given and interrupted sutures were left in place. (a) At day 8 after transplantation the graft is clear. (b) The same corneal graft 8 days after the onset of rejection, on day 18. The donor is opaque and vessels are invading the donor cornea.

were similar in matched and mismatched grafts (Fig. 3). As recipients possessed on average $50 \%$ of the haploid DA donor genome, this low (i.e. substantially less than $50 \%$ ) survival of matched grafts implies that several independently segregating non-MHC gene products are involved. The greater range of survival times of this matched group may reflect varying numbers of non-MHC mismatches. The rejection curves of matched and mismatched grafts in Fig. 2 are almost superimposed in the early stages after transplantation, suggesting that multiple non-MHC mismatches induce rejection as vigorously as MHC mismatches - a situation that parallels that observed with skin graft rejection. ${ }^{21}$ The potency of non-MHC gene products in stimulating an immune response may be enhanced in circumstances where donor and recipient are MHC-matched, because the recipient $\mathrm{T}$ cell repertoire has been specifically selected during development to recognise peptides presented in the context of self (in this case matched) MHC. Such a phenomenon may also account for the apparent detrimental effect of DR matching observed in the UK Corneal Transplant Follow-up Study ${ }^{10}$ (see below and Fig. 4).

The importance of non-MHC antigens in rodent corneal graft rejection has been confirmed by Sonoda and Streilein in a mouse study ${ }^{22}$ and is surprising in view of the so-called privileged status of the cornea. However, cornea and skin are derived from analogous embryonic tissues and both have a surface location. Once corneal privilege is abrogated by vascularisation and ingress of lymphatic vessels,

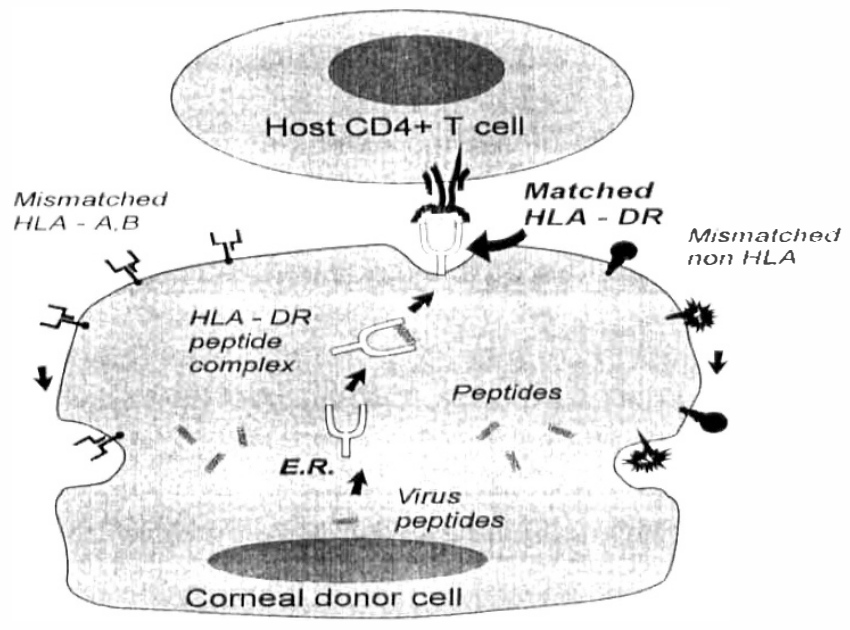

(a)

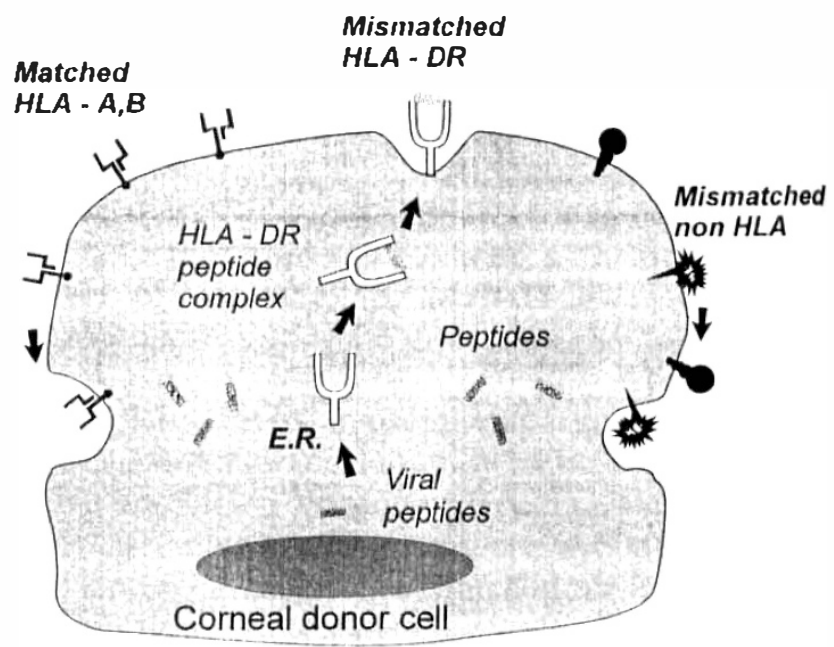

(b)

Fig. 4. Illustration of how a donor corneal cell might engage a host $C D 4^{+} T$ cell. (a) is rejection efficient because allogeneic peptides derived from mismatched genes (HLA-A,-B or non-MHC) or viruses can be presented to host $T$ cells on compatible $D R$ molecules. $(b)$ is rejection inefficient because $H L A-A,-B$ is matched and host $C D 4^{+} T$ cells are less able to engage due to incompatible HLA-DR molecules. E.R., endoplasmic reticulum. 
MHC mismatched

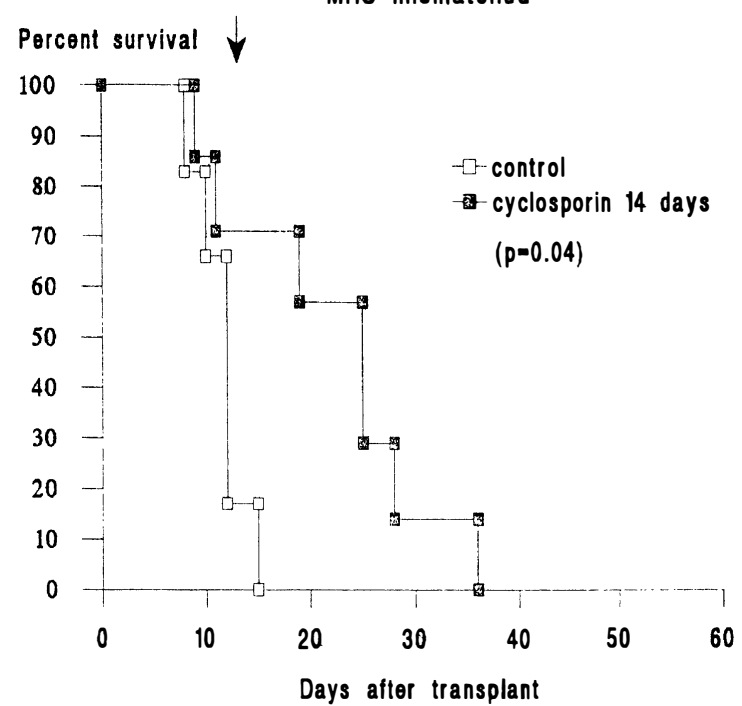

MHC mismatched
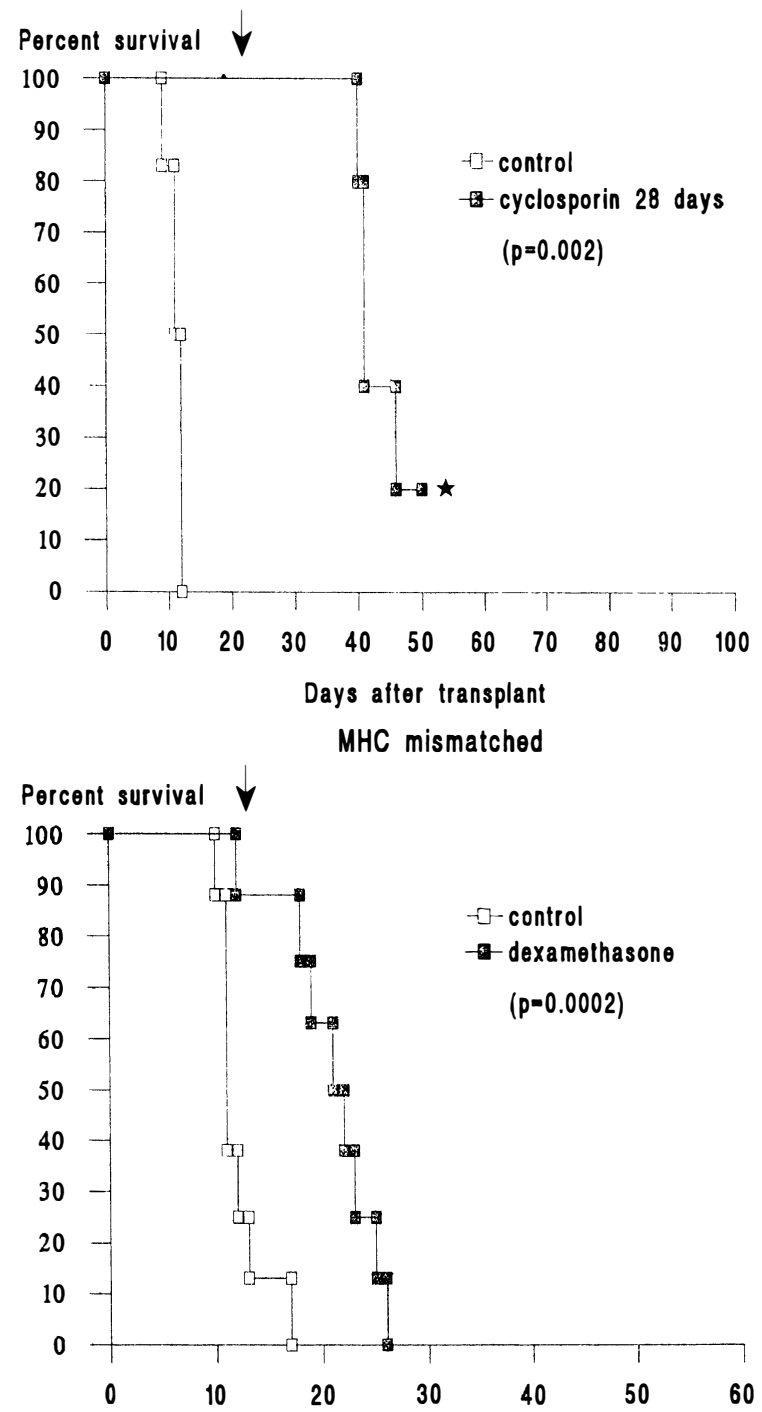

Days after transplant

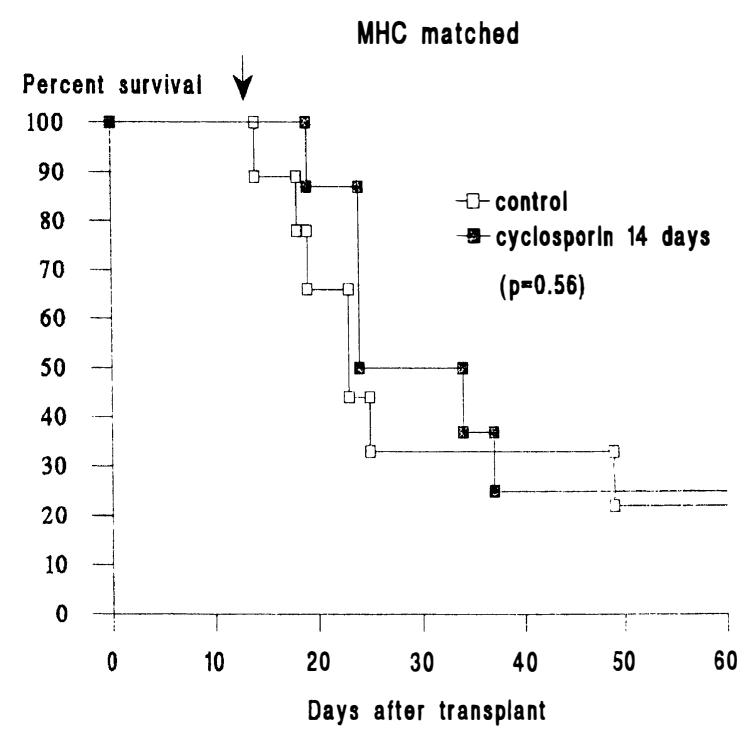

(a)

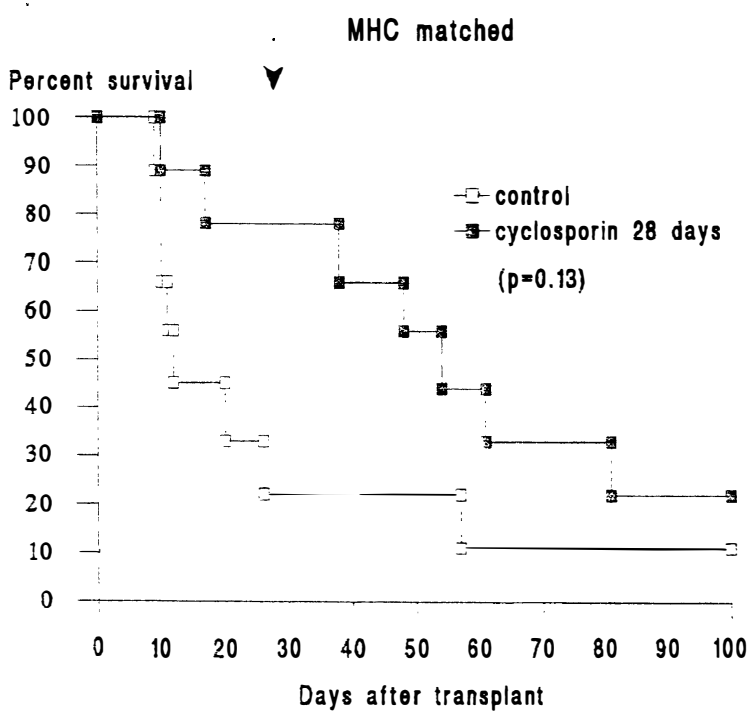

(b)

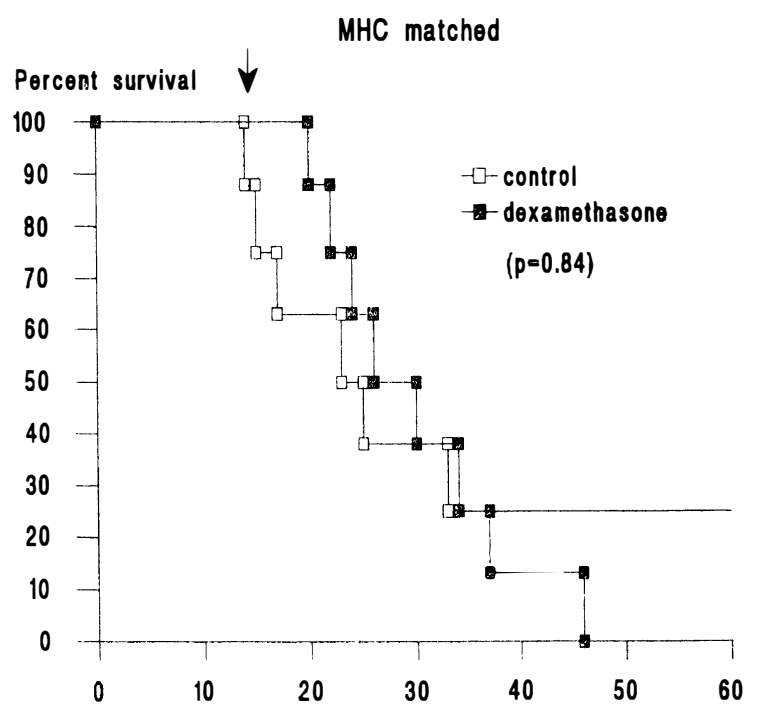

(c)

Fig. 5. Effect of systemic immunosuppressants on survival of MHC-matched and MHC-mismatched corneal grafts in the rat. Drugs were given from the day of transplantation, arrows indicating cessation of treatment. Control animals received drug vehicle. (a) Intraperitoneal injection of $10 \mathrm{mg} / \mathrm{kg}$ per day cyclosporin for 14 days; (b) the same treatment for 28 days; (c) $15 \mu \mathrm{l}$ $0.1 \%$ dexamethasone applied topically to the eye for 14 days. Immunosuppression significantly prolonged survival in the MHC-mismatched groups only (p values are shown). *Remaining animal died under anaesthesia. 
sensitisation of the recipient to corneal antigens would occur via the draining lymph nodes, as occurs with skin. Some animals in our backcross experiments were sensitised with female DA skin grafts prior to corneal transplantation. Sensitisation significantly reduced survival in matched as well as in mismatched groups, ${ }^{20}$ showing that the non-MHC antigens responsible for rejection are shared with skin. As in humans, the precise identity of non-MHC targets is unknown, with the possible exception of the $\mathrm{H}-\mathrm{Y}$ (male) antigen, which is expressed on cornea and can induce an immune response. ${ }^{23}$ This antigen was not involved in our experiments since all donors were female. In rats, erythrocyte antigens do not appear to be involved in rejection, ${ }^{24}$ at least in unsensitised animals, although some reports indicate that they are important in humans. ${ }^{9}, 25$

It should be noted that the recipients of MHCmatched grafts were all heterozygous $\left(\mathrm{RT}^{\mathrm{a} / 1}\right)$ at MHC loci. This heterozygosity might enhance the immune response by increasing the repertoire of donor peptides capable of presentation to $\mathrm{T}$ cells by recipient antigen presenting cells and is analogous to the human situation in that most humans are heterozygous at MHC loci.

We next addressed the question of whether MHCmatching would reduce the requirement for immunosuppression. Systemic immunosuppressants, although not routinely given after corneal transplantation, are sometimes necessary in high-risk cases and therefore both systemic cyclosporin and topical steroid were tested. As previously, $(\mathrm{DA} \times \mathrm{LEW}) \mathrm{F}_{1}$ $\times$ LEW recipients were tissue-typed and divided into groups that were either MHC-matched or mismatched with the DA donor. Equal proportions of males and females were included in each group. From the day of corneal transplantation one of three immunosuppressive treatments was given: (1) cyclosporin, $10 \mathrm{mg} / \mathrm{kg}$ per day for 14 days, (2) cyclosporin, $10 \mathrm{mg} / \mathrm{kg}$ per day for 28 days, or (3) $15 \mu \mathrm{l} 0.1 \%$ dexamethasone, topically to the transplanted eye, twice daily for 14 days. Control rats received vehicle alone. A further group of control rats $(7$ male and 4 female) received autografts followed by steroid treatment as above. Grafts were examined and scored as previously for opacity, oedema and vessel ingress. One autograft receiving dexamethasone developed a persistent epithelial defect during steroid treatment, accompanied by vascularisation and opacity and was killed. The remainder experienced transient oedema during the first week, then cleared and remained clear for the study period. As in our previous study, there was a high incidence of rejection of matched grafts. All three immunosuppressive regimes significantly delayed rejection in MHC-mismatched groups compared with controls (Fig. 5). However, in matched groups, although immunosuppression caused a trend towards delayed rejection, this was not significant, although blood levels of cyclosporin reached therapeutic levels (data not shown). Thus, the immune response to matched grafts appears to be relatively resistant to immunosuppression. The data confirm that the benefits of matching are less obvious with immunosuppressive therapy. This is not so much a result of the overall suppression of rejection, but because the response to non-MHC mismatches is less easily suppressed.

The resistance appeared to be a general effect in that it occurred with both topical and systemic treatment and with both agents. The phenomenon has previously been noted by Pinto et al. ${ }^{26}$ with respect to cyclosporin and skin graft rejection. RT1A (class I) mismatches were more easily suppressed than RT1B (class II) or RT1AB (class I and II) mismatches, while survival of MHC-matched grafts was not prolonged.

Further evidence of such resistance comes from a large multicentre international study on kidney transplants in man. In this study the well-documented improvement in survival of first and second graft afforded by the addition of cyclosporin to treatment regimes in the 1980s was not evident in the subgroup of patients where the donor was an HLA-identical sibling, i.e. where rejection was attributable only to non-MHC mismatches. ${ }^{27}$ Moreover, analysis of renal allograft failure in the United Kingdom from 1978 to 1988 showed that the beneficial effect of cyclosporin therapy disappeared 1 year after transplantation, as did the major benefit of HLA matching. ${ }^{28}$ This would be explicable if immunosuppression-resistant non-MHC antigens contribute disproportionately to late rejection.

The reasons for such resistance to immunosuppression are not clear. It is possible that non-MHC antigens are presented to the immune system by a different mechanism that is relatively resistant to suppression, e.g. indirectly via recipient antigen presenting cells rather than directly by dendritic cells of the donor. This notion is supported by the

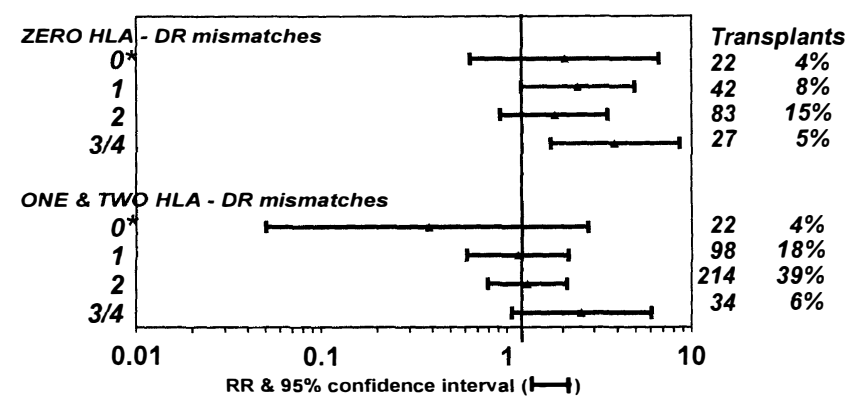

Fig. 6. Relative risk of rejection (triangle) plotted on a $\log _{10}$ scale for different levels (*) of HLA-A and -B mismatch, where donor and recipient are either matched or mismatched for DR. DR matching appears to prejudice graft survival. Bars represent $95 \%$ confidence intervals. 
recent findings of Sawyer et al. ${ }^{29}$ who have shown that peptide priming for indirect allorecognition of MHC class I antigens in rats results in alloantibody production that is resistant to suppression by cyclosporin. They suggest that the resistant cells are $\mathrm{T}$ cells involved in indirect help. Whatever the explanation for these observations, if a similar situation prevails in man, matching will unfortunately not permit a reduction in dosage of immunosuppressants.

At present there is no direct evidence in man that non-MHC antigens play a role in corneal graft rejection. However, careful clinical studies performed through the Corneal Transplant Follow-up Study in a series of 547 HLA-A, -B and -DR typed corneal transplants show that the relative risk of rejection increases with increasing mismatch for HLA-A and -B, but that HLA-DR mismatching appears to have no detrimental effect. Furthermore, the data suggest that HLA-DR matching is associated with an increased risk of rejection ${ }^{10}$ (Fig. 6). This observation can be explained in theory if HLADR molecules play a central role in allogeneic peptide presentation, where the latter are derived from HLA-A, -B, non-HLA or viral genes - i.e. as is proposed above for the rat, any mismatched gene products will be more efficiently presented where donor and recipient are matched at this locus (Fig. 4).

Careful multicentre matching studies and further animal investigations are required to elucidate these clinical and experimental findings. In particular we need to determine whether peptides derived from non-MHC mismatches are more efficiently presented where donor and recipient are partially or wholly MHC-matched.

Key words: Corneal transplantation, Cyclosporin, Graft rejection, Immunosuppression, Minor histocompatibility antigens, Rat.

\section{REFERENCES}

1. Allansmith MR, Fine M, Payne R. Histocompatibility typing and corneal transplantation. Trans Am Acad Ophthalmol Otolaryngol 1974;78:445-60.

2. Batchelor JR, Casey TA, Gibbs DC, et al. HLA matching and corneal grafting. Lancet 1976;1:551-4.

3. Stark WJ, Taylor HR, Bias WB, Maumenee E. Histocompatibility (HLA) antigens and keratoplasty. Am J Ophthalmol 1978;86:595-604.

4. Volker-Dieben HJ, Kok-van Alphen CC, D'Amaro J, de-Lange P. The effect of prospective HLA-A and -B matching in 288 penetrating keratoplasties for herpes simplex keratitis. Acta Ophthalmol (Copenh) 1984;62:513-23.

5. Boisjoly HM, Roy R, Dube I, et al. HLA-A, B and DR matching in corneal transplantation. Ophthalmology 1986;93:1290-7.

6. Sanfilippo F, MacQueen JM, Vaughn WK, Foulks GN. Reduced graft rejection with good HLA-A and -B matching in high-risk corneal transplantation. $\mathrm{N}$ Engl J Med 1986;315:29-35.

7. Boisjoly HM, Raynald R, Bernard PM, et al. Association between corneal allograft reactions and HLA compatibility. Ophthalmology 1990;97:1689-98.

8. Baggesen K, Ehlers N, Lamm LU. HLA-DR/RFLP compatible corneal grafts. Acta Ophthalmol (Copenh) 1991;69:229-33.

9. Collaborative Corneal Transplantation Studies Research Group. The Collaborative Corneal Transplantation Studies (CTTS). Effectiveness of histocompatibility matching in high-risk corneal transplantation. Arch Ophthalmol (Copenh) 1992;110:1392.

10. Vail A, Gore SM, Bradley BA, Easty DL, Rogers CA, Armitage WJ, on behalf of the Corneal Transplant Follow-up Study collaborators. Influence of donor and histocompatibility factors on corneal graft outcome. Transplantation (in press).

11. Fine M, Stein M. The role of corneal vascularisation in human corneal graft reactions. In: Porter R, Knight J, editors. Corneal graft failure. Ciba Foundation Symposium 15. Amsterdam: Elsevier, 1973:193-208.

12. Pleyer U, Steuhl KP, Weidle EG, et al. Corneal graft rejection: incidence, manifestation, and interaction of clinical subtypes. Transplant Proc 1992;4:2034-7.

13. Maumenee AE. Clinical patterns of corneal graft failure. In: Porter R, Knight J, editors. Corneal graft failure. Ciba Foundation Symposium 15. Amsterdam: Elsevier, 1973:5-23.

14. Williams KA, Coster DJ. Penetrating corneal transplantation in the inbred rat: a new model. Invest Ophthalmol Vis Sci 1985;26:23-30.

15. Katami M. Corneal transplantation: immunologically privileged status. Eye 1991;5:528-48.

16. Ross J, He Y-G, Niederkorn JY. Class I disparate corneal grafts enjoy afferent but not efferent blockade of the immune response. Curr Eye Res 1991;10:889-92.

17. Katami M, White DJG, Watson PG. An analysis of corneal graft rejection in the rat. Transplant Proc 1989;21:3147-9.

18. Katami M, Lim SML, Kamada N, et al. A pure class II MHC disparity does not induce rejection of cornea or heart grafts in the rat. Transplant Proc 1990;22:2200-1.

19. Ross J, Callanan D, Kunz H, Niederkorn J. Evidence that the fate of class II-disparate corneal grafts is determined by the timing of class II expression. Transplantation 1991;51:532-6.

20. Nicholls SM, Bradley BA, Easty DL. Effect of mismatches for major histocompatibility complex and minor antigens on corneal graft rejection. Invest Ophthalmol Vis Sci 1991;32:2729-34.

21. Graff RJ, Snell GD. Histocompatibility genes of mice. IX. The distribution of the alleles of the non-H-2 histocompatibility loci. Transplantation 1969;8:861-76.

22. Sonoda Y, Streilein JW. Orthotopic corneal transplantation in mice: evidence that the immunogenetic rules of rejection do not apply. Transplantation 1992;54: 694-704.

23. He Y-G, Ross J, Callanan D, Niederkorn JY. Acceptance of $\mathrm{H}-\mathrm{Y}$ disparate corneal grafts despite concomitant immunisation of the reicipient. Transplantation 1991;51:1258-62.

24. Katami M, Madden PW, White DJ, Watson PG, Kamada N. The extent of immunological privilege of orthotopic corneal grafts in the inbred rat. Transplantation 1989;48:371-6.

25. Allansmith MR, Daniel W, Drell BA, Kajiyema G. $\mathrm{ABO}$ blood groups and corneal transplantation. Am J Ophthalmol 1975;79:493-501. 
26. Pinto M, Gill TJ, Kunz HW. Prolongation of skin graft survival across different generic barriers in rats with cyclosporine: and its potentiation by Bordetella pertussis vaccine. Transplantation 1983;36:171-7.

27. Opelz G, for the Collaborative Transplant Study. Collaborative Transplant Study: 10 year report. Transplant Proc 1992;24:2342-55.

28. Bradley BA, Gilks WR, Gore SM. A preliminary analysis of late renal allograft failure. In: Terasaki PI, editor. Clinical transplants 1989. Los Angeles: UCLA Tissue Typing Laboratory, 1989;175-83.

29. Sawyer GJ, Dalchau R, Fabre JW. Indirect T cell allorecognition: a cyclosporin A resistant pathway for $\mathrm{T}$ cell help for antibody production to donor MHC antigens. Transplant Immunol 1993;1:77-81. 